\title{
Regulate e-cigarettes as drug-delivery devices
}

\author{
Matthew B. Stanbrook MD PhD
}

See also practice article by Reed and colleagues on page 1427 and at www.cmaj.ca/lookup/doi/10.1503/cmaj.130806

$\mathrm{A}$ ddiction now comes in its own "e" version: electronic cigarettes. By allowing the user to inhale a vapour in a manner resembling smoking a cigarette, e-cigarettes provide a uniquely appealing means of obtaining nicotine without exposure to the other harmful constituents of tobacco smoke. Their increasing popularity is paralleled by growing controversy over whether e-cigarettes represent a highly promising therapeutic intervention for smoking cessation or a Trojan horse that will allow the tobacco industry to reverse decades of global progress in reducing smoking prevalence.

Nicotine is a drug, and e-cigarettes containing nicotine are drug-delivery devices. Health Canada has always treated them as such, which makes Canada's laws governing these devices among the most restrictive in the world. Not all e-cigarettes contain nicotine, and selling non-nicotine e-cigarettes is legal in Canada. Some jurisdictions that currently allow e-cigarettes, such as the United Kingdom and the European Union, are planning to regulate them as drugs. ${ }^{1}$ However, in the United States, e-cigarettes have flourished in the virtual absence of regulatory controls. The US market has doubled in size every year since 2007 and now represents a \$1 billion industry. ${ }^{2}$ Consequently, even though Canadian regulations prohibit the sale, import or advertising of nicotine-containing e-cigarettes, ${ }^{3}$ they are surreptitiously crossing our border. The US Food and Drug Administration is poised to declare new regulations for e-cigarettes, but it will do so under the weaker rules that apply to tobacco products, instead of drug regulations.

A recent nationally representative US survey found that use of e-cigarettes doubled from 2011 to 2012 among middleschool and high-school students; $10 \%$ of users had never previously used tobacco. ${ }^{4}$ Less than half of US states currently restrict sales or marketing of e-cigarettes to minors. E-cigarette companies are thus free to tempt US youth (and those watching from abroad) to imitate smoking behaviour with fruitflavoured products and movie-star endorsements, and to resurrect marketing campaigns originally designed, but no longer permitted, for cigarettes. Fears therefore arise that a new generation of youth who would not otherwise take up smoking could be enticed by e-cigarettes into nicotine addiction and subsequent tobacco use.

Arguments in favour of e-cigarettes are predicated on the assumption that their availability will lead to cessation of tobacco use. They have theoretical advantages over existing forms of nicotine replacement therapy because they provide the experience of holding and inhaling from a cigarette-like device and allow users to regulate the nicotine dose by the way they inhale. A troubling alternative possibility, however, is that e- cigarettes will merely supplement tobacco use in contexts where smoking is no longer allowed and may thereby perpetuate smoking among people who would otherwise be motivated to continue trying to quit. The more recent of the 2 existing randomized trials of e-cigarettes as smoking cessation aids failed to show superiority over a nicotine patch for the primary outcome, continuous abstinence from smoking at 6 months. ${ }^{5}$ Despite having a free supply of e-cigarettes at hand, most of the participants continued to smoke cigarettes. Concerns are amplified further by the emergence of tobacco companies as major players in the e-cigarette industry without any accompanying slowdown in tobacco production or marketing, which suggests that the tobacco industry sees a future where e-cigarettes accompany and perpetuate, rather than supplant, tobacco use.

The most important interventions responsible for the decline in smoking prevalence have not been nicotine replacement or other pharmacotherapy, or the explicit and consistent advice of health professionals, but rather the concerted public health and public policy efforts that have gradually denormalized, restricted and marginalized smoking behaviour in much of the world over the last 40 years. For many smokers, only the inconvenient disincentives to smoking provided by these measures proved sufficient to counteract the addiction-based incentives that would otherwise drive them back to smoking. We must not be so easily lured by the illusion of a safe substitute for cigarettes that we yield precious ground in the war against tobacco.

It may be tempting to embrace e-cigarettes on the premise that they cannot be more dangerous than smoking cigarettes. Our decision-making must be guided by adequate scientific knowledge about the quality, efficacy, safety and unintended consequences of these products, just as it is for all other drugs and devices. Industry must be motivated to develop and test ecigarettes as pharmaceutical, rather than tobacco, products, but this will happen only if a sufficient number of countries representing a large share of the global market regulate them as such. Let us be glad that, in Canada at least, policy-makers appear to have learned that lesson from a century of bitter experience with tobacco.

For references, see Appendix 1, available at www.cmaj.ca/lookup/suppl/doi :10.1503/cmaj.131469/-/DC1.

Competing interests: See www.cmaj.ca/site/misc/cmaj_staff.xhtml

Affiliation: Matthew Stanbrook is Deputy Editor, CMAJ.

Correspondence to: $C M A J$ editor, pubs@cmaj.ca

CMAJ 2013. DOI:10.1503/cmaj.131469 